\title{
RENDIMENTO E QUALIDADE DE FRUTOS DE PIMENTÃO CULTIVADO EM AMBIENTE PROTEGIDO EM FUNÇÃO DO NITROGÊNIO E POTÁSSIO APLICADOS EM COBERTURA
} \author{
Henriqueta M. Gimenes Fernandes ${ }^{2}$; Walkyria Bueno Scivittaro ${ }^{4}$ \\ ${ }^{1}$ Universidade Estadual de Maringá, Av. Colombo 5790 - CEP: 87020-900 - Maringá, PR. \\ ${ }^{2}$ CENA/USP, C.P. 96 - CEP: 13400-970 - Piracicaba, SP. \\ ${ }^{3}$ Depto. de Fitotecnia - IAC, C.P. 28 - CEP: 13002-902 - Fazenda Santa Elisa - Campinas, SP. \\ ${ }^{4}$ Embrapa Clima Temperado, C.P. 403 - CEP: 96001-970 - Pelotas, RS. \\ *e-mail: magsilva@uem.br
}

Maria Anita Gonçalves da Silva ${ }^{1 *}$; Antonio Enedi Boaretto²; Arlete Marchi Tavares de Melo3;

RESUMO: $\mathrm{O}$ objetivo deste trabalho foi avaliar o efeito do $\mathrm{N}$ e $\mathrm{K}_{2} \mathrm{O}$ na produtividade e rendimento do pimentão, em função de características de crescimento dos frutos (peso, comprimento e diâmetro) e do número total de frutos por planta e por área. Os tratamentos foram aplicados sob condições de ambiente protegido, ao lado da linha de plantas e parcelados em 6 vezes, utilizando-se a cultivar Mayata. 0 experimento foi conduzido de novembro/96 a agosto/97 e constou dos seguintes tratamentos: controle e as combinações de 3 doses de $\mathrm{N}\left(13,3 ; 26,6\right.$ e $\left.39,9 \mathrm{~g} \mathrm{~m}^{-2}\right)$ e 3 doses de $\mathrm{K}_{2} \mathrm{O}\left(13,3 ; 26,6\right.$ e $\left.39,9 \mathrm{~g} \mathrm{~m}^{-2}\right)$. O delineamento experimental adotado foi o de blocos ao acaso, em esquema fatorial $(3 \times 3+1)$, com 4 repetições, totalizando 40 parcelas. Cada parcela foi composta de 11 plantas, totalizando 444 plantas. Foram realizados todos os tratos culturais recomendados para a cultura do pimentão, incluindo irrigação por gotejamento. Os frutos foram colhidos recém maduros, ao atingirem ponto de consumo. Com base nos resultados, concluiu-se que a adubação nitrogenada aumentou a produção total de matéria seca das plantas (caule, folhas e raízes), embora não tenha afetado a produção de matéria seca dos frutos. $O$ nível ótimo estimado de acúmulo de $\mathrm{M}$. seca ocorreu na dose de $27,0 \mathrm{~g} \mathrm{~m}^{-2}$ de $\mathrm{N}$. A aplicação de $\mathrm{N}$ no solo não influenciou significativamente, as características de crescimento dos frutos, como peso, comprimento e diâmetro, nem o rendimento de frutos comerciais; o efeito favorável do $\mathrm{N}$ na produção de matéria seca das plantas e rendimento dos frutos, ocorreu somente em baixas doses de $\mathrm{K}_{2} \mathrm{O}$ no solo $\left(13,3 \mathrm{~g} \mathrm{~m}^{-2}\right)$, aplicado como $\mathrm{KCl}$. As maiores doses de $\mathrm{K}_{2} \mathrm{O}$ tiveram um efeito depressivo para os mesmos parâmetros, havendo um mínimo de crescimento vegetativo com a dose estimada de $33,0 \mathrm{~g} \mathrm{~m}^{-2}$ de $\mathrm{K}_{2} \mathrm{O}$.

Palavras-chave: Capsicum annuum, nitrogênio, potássio, adubação, rendimento, pimentão

\section{FRUIT YIELD AND QUALITY OF SWEET PEPPER GROWN IN PROTECTED ENVIRONMENT, INFLUENCED BY NITROGEN AND POTASSIUM FERTILIZATION}

\begin{abstract}
ABASTRACT: The objective of this work was to evaluate the effect $\mathrm{N}$ and $\mathrm{K}_{2} \mathrm{O}$ on the production and yield of sweet pepper plants, related to the characteristics of growth (weight, lenght and diameter) and total number of fruits per plant, per area. Nitrogen (urea) and potassium (KCl) fertilizers were side dressed in six applications to sweet pepper cultivar Mayata, cultivated in a protected environment. The experiment was conducted from November/96 to August/97, with the following treatments: control and combination of three rates of $\mathrm{N}\left(13.3,26.6\right.$, and $\left.39.9 \mathrm{~g} \mathrm{~m}^{-2}\right)$ and three rates of $\mathrm{K}_{2} \mathrm{O}(13.3,26.6$ and $39.9 \mathrm{~g} \mathrm{~m}^{-2}$ ). The experimental design consisted of randomized blocks, in a factorial scheme $(3 \times 3+1)$, with four replications with a total of 40 plots. Each plot comprised 11 plants, totalizing of 444 plants. Plants were grown with drip irrigation along the entire cycle of the crop. Fruits were harvest when ripe, ready for consumption. Nitrogen fertilizer increased the maximun dry matter of the stem, leaves and roots, but not of fruit dry matter production. The optimal rate for maximum dry matter accumulation of $\mathrm{N}$ was $27.0 \mathrm{~g} \mathrm{~m}^{-2}$. Nitrogen did not affect the growth characteristics of the commercial fruits, such as weight, length, and diameter. Therefore did not affect the yield of commercial fruits. The favorable effect of the nitrogen on the production of total dry matter and yield occurred only at low concentrations of potassium in the soil $\left(13.3 \mathrm{gm}^{-2}\right)$ and a minimum development of sweet pepper with $33,0 \mathrm{~g} \mathrm{~m}^{-2} \mathrm{de} \mathrm{K}_{2} \mathrm{O}$ in the soil.
\end{abstract}

Key words: Capsicum annuum, nitrogen, potassium, fertilization, yield, sweet pepper 


\section{INTRODUÇÃO}

O pimentão destaca-se entre as solanaceas pelo seu consumo e importância econômica no Brasil e no exterior, principalmente nos Estados Unidos, México, Itália, Japão e Índia.

No mercado, os frutos de coloração verde e vermelha, são mais aceitos, embora aqueles de cor laranja, amarelo e até lilás, mais exóticos, têm alcançado bons preços, mais pela excentricidade. A pigmentação influencia no sabor e aroma, sendo que os frutos vermelhos são mais saborosos, porque apresentam $50 \%$ mais substância picante, a capsaína (Fonseca, 1986). O valor nutritivo do pimentão deve-se à presença de vitaminas, em especial a vitamina $\mathrm{C}$, essencial à nutrição humana, cujo teor pode chegar até $15 \mathrm{~g} \mathrm{~kg}^{-1}$ de peso seco, além de 10 $\%$ de proteinas (El Saied, 1995). Contém ainda vitaminas $\mathrm{A}, \mathrm{B} 1, \mathrm{~B} 2$ e minerais como $\mathrm{Ca}, \mathrm{Fe}$ e $\mathrm{P}$ (Poblete, 1971).

Atualmente o cultivo de hortaliças em ambiente protegido, entre as quais o pimentão, assume cada vez mais importância, porque permite a produção em diferentes épocas, alcançando maiores preços no mercado. Porém, no Brasil há escassez de informações sobre o comportamento e as exigências nutricionais da cultura nesse ambiente, sendo que, em geral, as recomendações de adubação se baseiam em trabalhos realizados com o pimentão, cultivado em condições de campo.

Um dos nutrientes mais limitantes para a cultura do pimentão é o nitrogênio, porque influencia no crescimento das plantas e produção dos frutos (Manchanda \& Singh, 1987; Shukla et al., 1987; Hochmuth et al., 1987; Manchanda \& Singh, 1988). Nas condições de cultivo da Flórida, EUA, utilizando-se níveis de 160 e $222 \mathrm{~kg} \mathrm{ha}^{-1}$ de $\mathrm{N}$, obteve-se, respectivamente, 80,7 e $101,7 \mathrm{~g}$ por planta de matéria seca nos frutos e 60,5 e $72,2 \mathrm{~g}$ por planta na produção da parte aérea (Hochmuth et al., 1987).

Foi observado por Gollifer (1993), na Índia, efeito do $\mathrm{K}$ no crescimento de plantas de pimentão, mesmo em solos ricos em matéria orgânica e de boa fertilidade. A maior produção de matéria seca, após 28 semanas do transplante foi de 102,$9 ; 76,3 ; 131,1$ e 24,1 (g por planta), respectivamente para caule, folhas, frutos $e$ raízes. Por outro lado, Chougule \& Mahajan (1979 ) não encontraram relação entre doses de $\mathrm{K}$ e crescimento da planta.

A influência do $\mathrm{N}$ e $\mathrm{K}$ no rendimento de frutos de pimentão foi estudada por vários autores (Iley \& Ozaki, 1966; Everett, 1976; Everett \& Subramanya, 1983). Avaliando a proporção N:K de 1:1,15, Everett (1976) encontrou melhores rendimentos quando altas quantidades dos nutrientes foram aplicadas no outono (doses de 726 e $837 \mathrm{~kg} \mathrm{ha}^{-1}$, respectivamente para $\mathrm{N}$ e K). Na primavera os melhores rendimentos exigiram a metade das doses utilizadas no outono. Segundo lley \& Ozaki (1966), altas quantidades de $\mathrm{K}$, comparativamente ao $\mathrm{N}$, em proporções de $1: 1 ; 1: 3 ; 1: 6$, estiveram relacionadas às produtividades de frutos. $\mathrm{O}$ nível 1 correspondeu a $336 \mathrm{~kg} \mathrm{ha}^{-1}$ de $\mathrm{N}$.

Quando a produção do pimentão foi avaliada através do número e peso médio dos frutos por planta, Mishriky \& Alphonse (1994) observaram que $0 \mathrm{~N}$ favoreceu esses parâmetros. De forma semelhante, Chougule \& Mahajan (1979) referem-se a um maior crescimento dos frutos em comprimento e diâmetro devido ao N. O K não afetou nenhum dos parâmetros avaliados.

Este trabalho foi executado com o

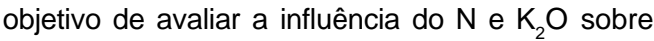
a produção de matéria seca total da planta, rendimento e características de frutos de pimentão, cultivado em ambiente protegido.

\section{MATERIAL E MÉTODOS}

O experimento foi realizado em ambiente protegido, utilizando a cultivar Mayata, em um latossolo vermelho escuro localizado no Núcleo Experimental do IAC, em Campinas.

A análise química, após calagem, adubação orgânica e fosfatada, apresentou na camada de 0 a $20 \mathrm{~cm}: \mathrm{pH}\left(\mathrm{CaCl}_{2}\right)=5,7$; matéria orgânica $=24 \mathrm{~g} \mathrm{dm}^{-3} ; \mathrm{P}$ (resina) $=199 \mathrm{mg} \mathrm{dm}^{-3}$; $\mathrm{S}-\mathrm{SO}_{4}=251 \mathrm{mg} \mathrm{dm}^{-3} ; \mathrm{N}$ total $=1,8 \mathrm{~g} \mathrm{~kg}^{-1}$; $\mathrm{K}=3,2 \mathrm{mmol}_{\mathrm{c}} \mathrm{dm}^{-3} ; \mathrm{Ca}=52 \mathrm{mmol}_{\mathrm{c}} \mathrm{dm}^{-3} ; \mathrm{Mg}=19$ $\mathrm{mmol}_{\mathrm{c}} \mathrm{dm}^{-3} ; \mathrm{H}^{+}+\mathrm{Al}^{3+}=22 \mathrm{mmol}_{\mathrm{c}} \mathrm{dm}^{-3} ; \mathrm{SB}=74$ $\mathrm{mmol}_{\mathrm{c}}^{\mathrm{c}} \mathrm{dm}^{-3} ; \mathrm{T}=96 \mathrm{mmol}_{\mathrm{c}} \mathrm{dm}^{-3} ; \mathrm{V}=77 \%$. Para a camada de 20 a $40 \mathrm{~cm}$, os resultados foram: $\mathrm{pH}$ $\left(\mathrm{CaCl}_{2}\right)=4,8$; matéria orgânica $=24 \mathrm{~g} \mathrm{dm}^{-3} ; \mathrm{P}$ (resina) $=39 \mathrm{mg} \mathrm{dm}^{-3} ; \mathrm{S}^{-\mathrm{SO}_{4}}=134 \mathrm{mg} \mathrm{dm}^{-3} ; \mathrm{N}$ total $=1,7 \mathrm{~g} \mathrm{~kg}^{-1} ; \mathrm{K}=3,5 \mathrm{mmol}^{-3} ; \mathrm{Ca}=27$ $\mathrm{mmol}_{\mathrm{c}} \mathrm{dm}^{-3} ; \mathrm{Mg}=14 \mathrm{mmol}_{\mathrm{c}} \mathrm{dm}^{-\beta} ; \mathrm{H}^{+}+\mathrm{Al}^{3+}=34$ $\mathrm{mmol}_{\mathrm{c}} \mathrm{dm}^{-3} ; \mathrm{SB}=45 \mathrm{mmol}_{\mathrm{c}} \mathrm{dm}^{-3} ; \mathrm{T}=79 \mathrm{mmol}_{\mathrm{c}}$ $\mathrm{dm}^{-3} ; \mathrm{V}=57 \%$.

O solo foi preparado com uma aração profunda, seguida de revolvimento com enxada rotativa e gradagem. A calagem foi feita em área total, baseada no resultado da análise química do solo, visando atingir uma saturação por bases igual a $80 \%$ para a camada de $0-20 \mathrm{~cm}$ de solo 
e elevar o teor de magnésio a um mínimo de 9 $\mathrm{mmol} \cdot \mathrm{dm}^{-3}$. Foram utilizadas 2,0 ton $\mathrm{ha}^{-1} \mathrm{de}$ calcário dolomítico, com $100 \%$ de PRNT. Seguindo-se a recomendação de Nagai (1995), incorporou-se adubo orgânico, numa quantidade relativa a $20 \mathrm{t} \mathrm{ha}^{-1}$.

A adubação mineral foi feita 7 dias antes do transplante das mudas, colocando-se 60 $\mathrm{g} \mathrm{m}^{-2}$ de $\mathrm{P}_{2} \mathrm{O}_{5}, 100 \mathrm{mg} \mathrm{m}^{-2}$ de $\mathrm{B}$ e $240 \mathrm{mg} \mathrm{m}^{-2}$ de $\mathrm{Zn}$, nas formas de superfosfato simples, bórax e sulfato de zinco, respectivamente. Os fertilizantes foram aplicados no sulco de plantio, seguindo recomendações de Boaretto (1986) e Raij et al. (1996).

Quando as mudas atingiram 50 dias de idade, em 01/11/96, foram transplantadas em sulcos com $10 \mathrm{~cm}$ de profundidade. As adubações nitrogenada (uréia) e potássica (cloreto de potássio) foram iniciadas 13 dias após o transplantio, em cobertura e parceladas em seis vezes, entre o período de 13/11/96 a 24/01/97. Os tratamentos compreenderam uma testemunha sem $\mathrm{N}$ e $\mathrm{K}_{2} \mathrm{O}$ e as combinações de três doses de nitrogênio $\left(13,3 ; 26,6\right.$ e $39,9 \mathrm{~g} \mathrm{~m}^{-2}$ de $\left.\mathrm{N}\right)$, e três doses de potássio (13,3; 26,6 e $39,9 \mathrm{~g} \mathrm{~m}^{-2}$ de $\mathrm{K}_{2} \mathrm{O}$ ). Os níveis intermediários de $\mathrm{N}$ e $\mathrm{K}_{2} \mathrm{O}$ foram escolhidos de acordo com resultados da análise do solo, seguindo recomendação de Boaretto (1986) e Raij et al. (1996). Os adubos foram aplicados ao lado das plantas, nas duas linhas de plantio, logo abaixo dos gotejadores.

O delineamento experimental adotado foi o de blocos ao acaso, em esquema fatorial $(3 \times 3+1)$, com 4 repetições, totalizando 40 parcelas. Cada parcela experimental foi composta de 11 plantas, dispostas em duas linhas num total de 444 plantas. Foram consideradas úteis as sete plantas em posição central, ocupando, cada planta, uma área de $0,30 \mathrm{~m}^{2}$, para $0,50 \mathrm{~m}$ entre plantas e 0,60 $\mathrm{m}$ entre linhas.

Durante todo o período do experimento foram realizados tratos culturais, como irrigação por gotejamento, capinas, desbrotas, podas de ramos e galhos não produtivos, tutoramento e tratamento fitossanitário.

Para a irrigação utilizaram-se fileiras de gotejadores colocados ao lado das linhas de plantio. O volume de água esteve entre a umidade mínima necessária para o pimentão e a umidade na capacidade de campo, considerando a área da parcela e a profundidade do sistema radicular. O volume de água mínimo limitante para o pimentão, para um potencial matricial (y) igual a $11 \mathrm{kPa}$, foi calculado na curva de retenção de água pelo solo (Wosten \&
Genuchten, 1988). A capacidade máxima de retenção de água foi obtida experimentalmente, de acordo com Pereira (1995). O tempo de irrigação das parcelas foi calculado em função da vazão dos gotejadores e do volume de água necessário. 0 potencial matricial, correspondente às umidades, foi lido em tensiômetros instalados no solo, nas profundidades de 20 e $40 \mathrm{~cm}$, no início, meio e final da estufa. Os cálculos do potencial matricial, correspondente a umidade na capacidade de campo, seguiram orientação de Wosten \& Genuchten (1988).

Durante um período de 34 semanas, entre 07/01/97 até 30/08/97, colheram-se os frutos que atingiram o ponto de consumo, totalizando 18 colheitas.

Em cada colheita foram avaliadas características de produção como peso de matéria fresca, comprimento e diâmetro em 3332 frutos, classificados como comerciais. Foram considerados frutos comerciais àqueles com coloração verde intenso a ligeiramente maduros, peso superior a $120 \mathrm{~g}$ e isentos de danos mecânicos e fisiológicos. Em quatro épocas de colheita $\left(2^{\mathrm{a}}, 6^{\mathrm{a}}, 10^{\mathrm{a}}\right.$ e $\left.16^{\mathrm{a}}\right)$, foram separadas amostras de 3 a 5 frutos por parcela, para determinação da matéria seca.

Ao final do experimento, 34 semanas após transplante, foram colhidas 7 plantas por parcela, totalizando 280 plantas. As plantas foram separadas em folhas com pecíolos, caules e frutos verdes, não comerciáveis e raízes. As partes da planta foram lavadas em água deionizada e secas em estufa a $65^{\circ} \mathrm{C}$ até peso constante, para a determinação da matéria seca. Os frutos não comerciáveis, ou seja, frutos verdes, com peso menor que $120 \mathrm{~g}$, queimados pelo sol, ou deformados, bem como os frutos descartados durante as desbrotas foram separados, contados e considerados como número total de frutos.

Para a coleta das raízes adotou-se a metodologia de Bohn (1979), com algumas modificações introduzidas pelo Centro de Ecofisiologia e Biofísica do IAC. Utilizou-se trado específico, com volume de $407 \mathrm{~cm}^{3}$. O peso do solo úmido correspondeu em média a $500 \mathrm{~g}$, com umidade entre 25 a $30 \%$, dependendo do dia de coleta. As raízes foram coletadas logo abaixo da linha de gotejo, nas profundidades de 0-10, 1020 e $20-40 \mathrm{~cm}$. Foram feitas 2 amostragens por planta, utilizando-se 3 plantas por parcela. 0 trado foi colocado a uma distância de $10 \mathrm{~cm}$ do caule da planta. Os tratamentos amostrados foram aqueles onde o menor nível de $\mathrm{N}$ estava combinado com o menor e com o maior nível de 
$\mathrm{K}_{2} \mathrm{O}$, bem como a combinação entre as doses máximas dos nutrientes. $\mathrm{O}$ mesmo procedimento foi adotado para a escolha dos tratamentos com níveis de $\mathrm{K}_{2} \mathrm{O}$, além do controle. O objetivo destes tratamentos, foi verificar a limitação no crescimento das raízes por um possível efeito de salinidade do sal $\mathrm{KCl}$, para altos níveis de $\mathrm{K}_{2} \mathrm{O}$ adicionado. A separação das raízes do solo foi feita por meio de dispersão em água e fracionamento do solo e pela suspensão e peneiramento das raízes (Fujimura et al., 1994). As raízes foram lavadas e colocadas para secar à sombra sobre papel toalha. Após a secagem fez-se a separação das impurezas, tais como raízes de ervas daninhas e material orgânico. A seguir, o material foi seco em estufa a $65^{\circ} \mathrm{C}$, por 48 horas, e determinou-se então o peso da matéria seca.

Foi utilizado o teste de Dunnett bilateral ao nível de $5 \%$ de probabilidade para comparar a testemunha com os demais tratamentos. Para a análise do crescimento das raízes, utilizou-se fatorial $2 \times 2$ e para a análise da matéria seca da parte aérea, crescimento e rendimento dos frutos, utilizou-se fatorial $3 \times 3$. Os graus de liberdade referentes aos níveis de $\mathrm{N}_{\text {e }} \mathrm{K}_{2} \mathrm{O}$ foram decompostos em polinômios ortogonais para obtenção da melhor equação de ajuste dos resultados (Gomes,1987).

\section{RESULTADOS E DISCUSSÃO}

\section{Produção de matéria seca}

$\mathrm{O} N$ teve efeito significativo sobre a produção da matéria seca do caule $(p<0,08)$, matéria seca das folhas $(p<0,05)$ e matéria seca do caule + folhas $(p<0,05)$, como observado na TABELA 1. $O$ efeito do $N$ foi linear e positivo, comparativamente ao efeito do $\mathrm{K}_{2} \mathrm{O}$ (TABELA 2), que apresentou um efeito depressivo para os mesmos parâmetros. Porém os nutrientes não influenciaram o crescimento dos frutos e da parte aérea.

De acordo com Malavolta et al. (1997) o $\mathrm{N}$ estimula a formação e o desenvolvimento de gemas floríferas e frutíferas, assim como a vegetação, porque faz parte da constituição de enzimas, coenzimas, vitaminas e proteinas que participam da absorção iônica, fotossíntese, respiração, multiplicação e diferenciação celular.

TABELA 1 - Produção de matéria seca das plantas de pimentão em resposta às doses de $\mathrm{N}$ aplicadas.

\begin{tabular}{|c|c|c|}
\hline M.S. na planta & Equação & $\mathrm{R}^{2}$ \\
\hline M.S. Caule & $Y=0,230892+0,00120 x$ & 0,82 \\
\hline M.S. Folha & $Y=0,110478+0,0008 x$ & 0,72 \\
\hline $\begin{array}{l}\text { M.S. Caule + } \\
\text { Folha }\end{array}$ & $Y=0,341356+0.020 x$ & 0,78 \\
\hline
\end{tabular}

O potássio quando aplicado no solo como $\mathrm{KCl}$, em altas concentações, aumenta a pressão osmótica e há diminuição do potencial osmótico da água do solo (Marschner, 1985). O efeito do cloreto, diminuindo a produção de matéria seca das plantas de pimentão, foi também relatado por Guines et al. (1996).

Observou-se uma interação significativa entre as doses de $\mathrm{N}$ e $\mathrm{K}_{2} \mathrm{O}$ na produção de matéria seca das partes vegetativas da planta (Figura 1). $\mathrm{O}$ efeito favorável do $\mathrm{N}$ no crescimento vegetativo das plantas ocorreu até a dose de $26,6 \mathrm{~g} \mathrm{~m}^{-2}$, porém, somente quando $\circ \mathrm{K}_{2} \mathrm{O}$ foi aplicado na menor dose. Nesse sentido, o potássio diminuiu a produção de matéria seca do caule e folhas, quando aplicado em doses acima de $13,3 \mathrm{~g} \mathrm{~m}^{-2}$. Doses de $\mathrm{N}$ estimadas acima de $27,0 \mathrm{~g} \mathrm{~m}^{-2}$ provavelmente causaram uma inibição competitiva entre $\circ \mathrm{NH}_{4}^{+}$e a absorção de outros cátions, como $\mathrm{K}^{+}, \mathrm{Ca}^{2+}$ e $\mathrm{Mg}^{2+}$, os quais desempenham funções específicas na planta. $O$ potássio está relacionado ao acúmulo e translocação de carboidratos e às menores perdas de água pela planta, porque regula a abertura e fechamento dos estômatos. $\mathrm{O} \mathrm{Ca}$ possue função estrutural, como integrante da parede celular, facilitando a absorção iônica, enquanto o $\mathrm{Mg}$ tem como principal função a de ativador enzimático, nos processo respiratórios $\mathrm{e}$ fotossintéticos.

TABELA 2 - Produção de matéria seca das plantas de pimentão em resposta às doses de $\mathrm{K}_{2} \mathrm{O}$ aplicadas.

\begin{tabular}{lcc}
\hline M.S. na planta & \multicolumn{1}{c}{ Equação } & $R^{2}$ \\
\hline M.S. Caule & $Y=0,509142-0,0174265 x+0,00026328 x^{2}$ & 1,00 \\
M.S. Folha & $Y=0,193694-0,0023179 x$ & 0,91 \\
M.S. Caule + Folha & $Y=0,760367-0,0249271 x+0,00036056 x^{2}$ & 1,00 \\
\hline
\end{tabular}




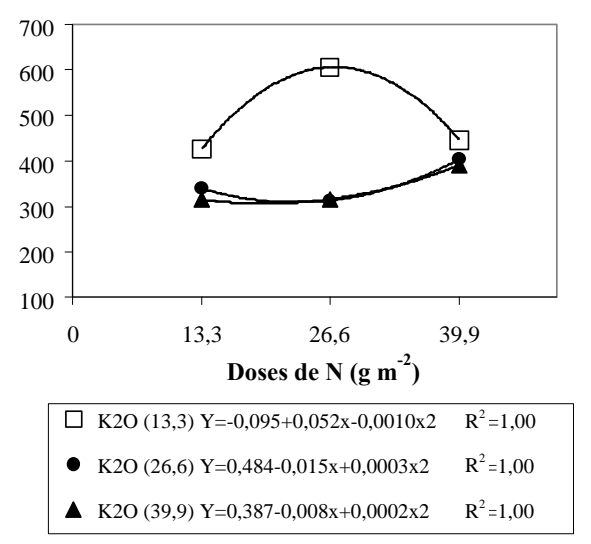

Figura 1 - Produção de matéria seca da parte vegetativa do pimentão $(\mathrm{C}+\mathrm{F})$ em função das doses de $\mathrm{N}$ no solo

Observou-se que o $\mathrm{K}$ diminuiu a produção da matéria seca do caule e folhas, principalmente quando o $\mathrm{N}$ foi aplicado na dose intermediária, uma vez que nessa dose obteve-se o maior crescimento da parte vegetativa da planta (Figura 2). A dose correspondente a um mínimo estimado de produção vegetativa foi de $33,0 \mathrm{~g} \mathrm{~m}^{-2}$ de $\mathrm{K}_{2} \mathrm{O}$.

Os relatos da literatura a respeito dos efeitos do $\mathrm{N}$ e $\mathrm{K}$ na produção de matéria seca de pimentão foram diferentes, em função das condições ambientais como clima e solo, como é verificado a seguir. Mishriki \& Alphounse (1994) constataram que as doses de $\mathrm{N}$ aumentaram a produção da parte vegetativa das plantas de pimentão, durante dois anos de experimentação, que aumentou de 75,9 a $89,4 \mathrm{~g}$ por planta, enquanto que Singh \& Srivastava (1988) encontraram menor produção de matéria seca da parte aérea, quando o $\mathrm{N}$ aumentou de 60 até $120 \mathrm{~kg} \mathrm{ha}^{-1}$ e Locascio et al. (1981) não encontraram resposta ao $\mathrm{N}$ na produção de matéria seca da parte aérea, com quantidades maiores que $224 \mathrm{~kg} \mathrm{ha}^{-1}$ de $\mathrm{N}$.

Trabalhando com $\mathrm{K}$ em solos da Flórida, Hochmuth et al. (1988) obtiveram em Martin, uma relação linear entre o peso de matéria seca da planta, e K aplicado ao solo. Níveis mais altos do nutriente aumentaram o tamanho da planta , mas não do fruto. Em outros 3 locais, não houve relação entre o $\mathrm{K}$ e matéria seca da planta e dos frutos.

O efeito favorável do $\mathrm{N}$ na produção de matéria seca de frutos foi reportado por Knavel (1977) e Olsen et al. (1993). Os últimos autores constataram aumentos de 140 para $178 \mathrm{~g}_{\text {fruto }}{ }^{-1}$

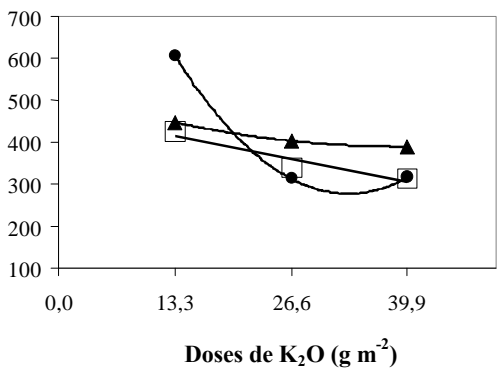

$$
\begin{array}{ll}
\square \mathrm{N}(13,3) \mathrm{Y}=0,470-0,0041 \mathrm{x} & \mathrm{R}^{2}=0,91 \\
\text { - } \mathrm{N}(26,6) \mathrm{Y}=1,192-0,055 \mathrm{x}+0,00083 \mathrm{x} 2 & \mathrm{R}^{2}=1,00 \\
\boldsymbol{\Delta} \mathrm{N}(39,9) \mathrm{Y}=0,519-0,007 \mathrm{x}+0,00008 \mathrm{x} 2 & \mathrm{R}^{2}=1,00
\end{array}
$$

Figura 2 - Produção de matéria seca da parte vegetativa do pimentão $(\mathrm{C}+\mathrm{F})$ em função das doses de $\mathrm{K}_{2} \mathrm{O}$ no solo

e 162 para $219 \mathrm{~g}_{\text {fruto }}{ }^{-1}$, repectivamente para o cultivo na primavera e outono, não concordando com os resultados obtidos por Locascio et al. (1981).

Miller et al. (1979), trabalhando com a cultivar Keystone Giant Resistant atribuíram, para $511 \mathrm{~kg} \mathrm{ha}^{-1}$ de matéria seca total produzida, um percentual de 30, 27 e 43, respectivamente para a produção da matéria seca do caule, folha e fruto. Rincon et al. (1995), em ambiente protegido, cultivar Lamuyo, constataram ao final do ciclo da cultura, após 21 semanas do transplante das mudas uma produção de $1,17 \mathrm{~kg}$ $\mathrm{m}^{-2}$ de matéria seca total (parte aérea), contribuindo os frutos em $67 \%$, as folhas em $19 \%$ e caules, em $14 \%$.

Os resultados de produção de matéria seca das raízes estão na TABELA 3. Em todas as profundidades amostradas, houve maior produção de raízes nos tratamentos com $\mathrm{N}$, sendo que $0 \quad \mathrm{~K}_{2} \mathrm{O}$ diminui este efeito. Provavelmente doses acima de $13,3 \mathrm{~g} \mathrm{~m}^{-2}$ de $\mathrm{K}_{2} \mathrm{O}$, aplicado como $\mathrm{KCl}$ diminuiu o crescimento das raízes em função do efeito salino do adubo que pode causar extravasamento do conteúdo celular nas raízes, prejudicando o seu crescimento. De acordo com Cruciani (1980) , o pimentão é uma cultura moderadamente sensível à salinidade.

Singh \& Srivastawa (1988), também encontraram efeito favorável do $\mathrm{N}$ na produção de raízes de pimentão, que aumentou de $6,5 \mathrm{~g}$ por planta para $10,1 \mathrm{~g}$ por planta.

Bar-Yosef et al. (1980) encontraram respostas favoráveis ao $\mathrm{N}$ e $\mathrm{K}$ na produção de raízes e relatam sua distribuição no solo, 
TABELA 3 - Produção de matéria seca de raízes em relação à testemunha .

\begin{tabular}{ccccc}
\hline & & \multicolumn{3}{c}{ Profundidade da amostra do solo $(\mathrm{cm})$} \\
\hline $\begin{array}{c}\mathrm{N} \mathrm{K}_{2} \mathrm{O} \\
\mathrm{g} \mathrm{m}^{-2}\end{array}$ & $0-10$ & $\begin{array}{c}10-20 \\
\text { por amostragem }\end{array}$ & $20-40$ \\
\hline 0,0 & 0,0 & 0,37 & 0,22 & 0,04 \\
13,3 & 13,3 & $0,59^{\star *(1)}$ & $0,42^{* *}$ & $0,12^{* *}$ \\
13,3 & 39,9 & $0,45 \mathrm{~ns}^{(2)}$ & $0,26 \mathrm{~ns}$ & $0,05 \mathrm{~ns}$ \\
39,9 & 13,3 & $0,54^{*(1)}$ & $0,40^{* *}$ & $0,11^{*}$ \\
39,9 & 39,9 & $0,49 \mathrm{~ns}$ & $0,27 \mathrm{~ns}$ & $0,09^{*}$ \\
\hline Média & & 0,49 & 0,31 & 0,08 \\
C V(\%) & 17 & 17 & 31 \\
\hline
\end{tabular}

(1) Diferenças significativas a $5 \%\left(^{\star}\right)$ e $1 \%\left(^{* \star}\right)$ de probabilidade ou ${ }^{(2)}$ não significativas, pelo teste de Dunnett, entre as médias dos tratamentos em relação à testemunha.

lateralmente à linha de gotejadores e em profundidade no perfil do solo. O N também influenciou favoravelmente o número e comprimento de raízes basais e laterais (Leskovar et al., 1989), entretanto Goyal et al. (1988) referem-se apenas a profundidade de amostragem afetando a massa radicular do pimentão.

\section{Rendimento e características dos frutos}

Os tratamentos que receberam as doses intermediárias de $\mathrm{N}\left(26,6 \mathrm{~g} \mathrm{~m}^{-2}\right)$ e a menor de $\mathrm{K}_{2} \mathrm{O}$ $\left(13,3 \mathrm{~g} \mathrm{~m}^{-2}\right)$ diferiram significativamente em relação à testemunha, quanto ao número total de frutos (frutos verdes e com peso menor que $120 \mathrm{~g}$ ), por área (Figura 3). Porém os tratamentos não influenciaram o número de frutos comerciais por área, (frutos recém maduros e com peso maior que $120 \mathrm{~g}$ ), assim como o rendimento dos frutos. Possivelmente a matéria orgânica presente no solo tenha diminuido o efeito do $\mathrm{N}$ aplicado através do fertilizante ou o manejo da cultura (podas), não foi efetuado corretamente, para que houvesse produção de frutos de maior tamanho ou peso.

$\mathrm{O}$ efeito favorável do $\mathrm{N}$ no rendimento de frutos de pimentão foi relatado por muitos autores (Locascio et al., 1981; Locascio \& Alligood, 1992; Locascio et al., 1985). Os rendimentos chegaram até $26,4 \mathrm{t} \mathrm{ha}^{-1} \mathrm{com}$ a aplicação máxima de $224 \mathrm{~kg}^{\mathrm{ha}}{ }^{-1}$ de N. Batal \& Smittle (1981), utilizando as cultivares Keystone Giant Resistant, e Hartz et al. (1993), referemse a rendimentos semelhantes aos citados, com a aplicação de 110 e $152 \mathrm{~kg} \mathrm{ha}^{-1}$ de $\mathrm{N}$, respectivamente. Também há relatos na literatura onde não foram encontradas respostas no aumento de produtividade, devido ao nutriente, como os de O' Sullivan (1979), na Flórida, durante três anos de experimentação, em solo franco arenoso com baixo teor de N. As cultivares estudadas foram Staddon's Select e Keystone Giant Resistant. Da mesma forma, Srinivas \& Prabhakar (1982) não encontraram efeito do $\mathrm{N}$ no número de frutos e produção de pimentão.

Há alguns relatos na literatura onde o $\mathrm{N}$ e $\mathrm{K}$ foram estudados conjuntamente, como Ozaki et al. (1955) e Olsen \& Lyons (1994). Da mesma forma, Moreno et al. (1996), em ambiente protegido, obtiveram um máximo rendimento de

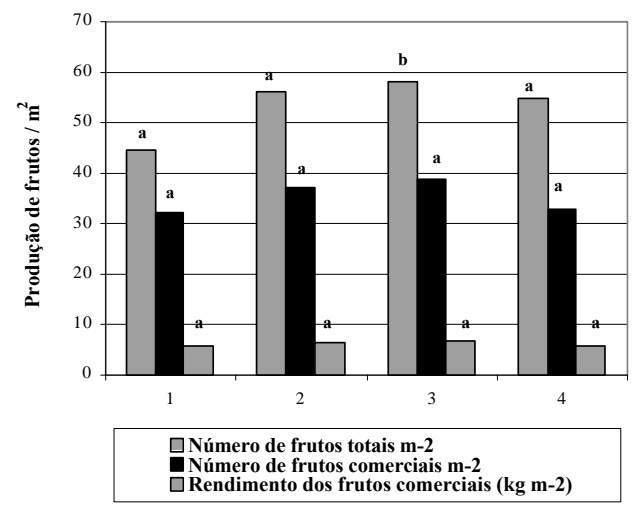

Figura 3 - Produção de frutos de pimentão em função das combinações de doses de $\mathrm{N}$ e $\mathrm{K}_{2} \mathrm{O}$ (1- ausência de $\mathrm{N}$ e $\mathrm{K}_{2} \mathrm{O} ; 2-\mathrm{N}=13,3$ e $\mathrm{K}_{2} \mathrm{O}=13,3 \mathrm{~g} \mathrm{~m}^{-2}$; $3^{2}-\mathrm{N}=26,6$ e $\mathrm{K}_{2} \mathrm{O}=13,3 \mathrm{~g} \mathrm{~m}^{-2}$; $4-\mathrm{N}=39,9$ e $\mathrm{K}_{2} \mathrm{O}=13,3 \mathrm{~g} \mathrm{~m}^{-2}$ ). Barras de mesmo padrão, com letras iguais não diferem entre si pelo teste de Dunnett a $5 \%$ de probabilidade. 
frutos total e comercial, com doses maiores de $\mathrm{N}$ e baixa quantidade de $\mathrm{K}$ aplicado, atribuindo os resultados ao maior número de frutos por planta. Nesse caso também, altas doses de $\mathrm{K}$ tiveram um efeito depressivo na produtividade, resultando em maior produção de frutos de segunda classe. A cultivar utilizada foi a Lamuyo.

Quanto à influência da adubação nitrogenada e potássica nas características dos frutos, os relatos da literatura são divergentes. Hochmuth et al. (1987), em 4 locais da Flórida, não encontraram efeito do $\mathrm{N}$ sobre o peso médio dos frutos, quando utilizaram as cultivares Jupiter e Early California Wonder. Por outro lado, Subhani et al. (1990) e Vanangamudi et al. (1990) referem-se a um efeito favorável dos nutrientes no comprimento e diâmetro dos frutos. Singh et al. (1988) obtiveram frutos com comprimento de $10,7 \mathrm{~cm}$ e diâmetro de 9,4 $\mathrm{cm}$, quando o solo recebeu $150 \mathrm{~kg} \mathrm{ha}^{-1} \mathrm{de}$ N. Hassan \& Ramlan (1994) referem-se a um maior número de frutos devido à adubação potássica.

A diversidade de resultados dá-se por conta da diversidade de condições em que os experimentos relatados na literatura foram desenvolvidos. Comparando-se a produtividade obtida no presente experimento, em média $6,1 \mathrm{~kg} \mathrm{~m} \mathrm{~m}^{2}$, com a obtida pelos autores acima citados, verificou-se um ótimo rendimento de frutos, mesmo no tratamento testemunha. Isso quer dizer que o solo, mesmo sem a adubação química, conseguiu suprir adequadamente as exigências das plantas de pimentão. As características dos frutos variam conforme a cultivar utilizado, entretanto as obtidas no presente experimento estão compatíveis com as citadas na literatura.

\section{CONCLUSÕES}

Para as condições que esse trabalho foi realizado, pode-se concluir que:

- A adubação nitrogenada aumentou a produção de matéria seca das plantas de pimentão até a dose máxima estimada de $27,0 \mathrm{~g} \mathrm{~m}^{-2}$, assim como aumentou também a produção de raízes. A produção de frutos não foi influenciada pela adubação nitrogenada.

- A aplicação de $\mathrm{N}$ no solo não influenciou as características de crescimento dos frutos, como peso, comprimento e diâmetro, bem como o rendimento de frutos comerciais.
- O efeito favorável do $\mathrm{N}$ na produção de matéria seca da parte vegetativa e raízes ocorreu somente em baixas concentrações de $\mathrm{K}_{2} \mathrm{O}$ no solo (13,3 $\mathrm{g} \mathrm{m}^{-2}$ ), aplicado como $\mathrm{KCl}$.

\section{REFERÊNCIAS BIBLIOGRÁFICAS}

BAR-YOSEF, B.; STAMMERS, C.; SAGIV, B. Growth of trickle irrigated tomato as related to rooting volume and uptake of $\mathrm{N}$ and water. Agronomy Journal, v.72, p.815-822, 1980.

BATAL, K.M.; SMITTLE, D. A . Response of bell pepper to irrigation, nitrogen and plant population. Journal American Society for Horticultural Science, v.106, n.3, p.259-262, 1981.

BOARETTO, A.E. Análise química de terra e recomendação de calagem e adubação para as principais culturas do Estado de São Paulo. Botucatu: Fundação de Estudos Agrícolas e Florestais, 1986. 50p. (Boletim Didático, 2).

BOHN, W. Methods of studying root systems. Berlin: Springer-Velag, 1979. 189p.

CHOUGULE, A B.; MAHAJAN, P.R. Effects of varying levels of plant population, nitrogen, phosphorus and potash on growth and yield of chilli (Capsicum annuum L.). Vegetable Science, v.6, n.2, p.73-80, 1979.

CRUCIANI, D.E. Adrenagem na agricultura. São Paulo: Nobel, 1980. 333p.

EI SAIED, H.M. Chemical composition of sweet and hot pepper fruits grown under plastic house conditions. Egyptian Journal of Horticulture, v.22, n.1, p.11-18, 1995.

EVERETT, P.H. Effect of nitrogen and potassium rates on fruit yield and size of mulch grown staked tomatoes. Proceedings Florida State of Horticultural Society, v.89, p.159-162, 1976.

EVERETT, P.H.; SUBRAMANYA, R. Pepper production as influenced by plant spacing and nitrogen-potassium rates. Proceedings Florida State of Horticultural Society, v.96, p.79-82, 1983.

FONSECA, A.F.A. da. Avaliação do comportamento de cultivares de pimentão (Capsicum annuum L.) em Rondônia. Porto Velho: EMBRAPA, 1986. 6p.

FUJIMURA, M.; KURACHI, S.A.H.; ARRUDA, F.B.; PIRES, R.C. de M. A técnica de estudo de raízes pelo método do trado. Campinas: IAC, 1994. 10p. (Boletim Técnico, 153).

GUINES, A.; INAL, A.; ALPASLAN, M. Effect of salinity on stomatal resistance, proline and mineral composition of pepper. Journal of Plant Nutrition, v.19, n.2, p.389-396, 1996. 
GOLLIFER, D.E. Effects of applications of mulch and potassium on Capsicum annuum. Papua New Guinea Journal of Agriculture, Forestry and Fisheries, v.36, n.1, p.22-29, 1993.

GOMES, F.P. A estatística moderna na pesquisa agropecuária. 2. ed. Piracicaba: Potafos, 1987. $162 p$.

GOYAL, M.R.; CRESPO RUIZ, M.; RIVERA, LE. Root distribution of nitrogen fertigated sweet peppers under drip irrigation. Journal of Agriculture of the University of Puerto Rico, v.72, n.1, p.51-55, 1988.

HARTZ, T.K.; LeSTRANGE, M.; MAY, D.M. Nitrogen requirements of drip-irrigated peppers. Hortscience, v.28, n.11, p.1097-1099, 1993.

HASSAN S.A.; RAMLAN, Z. A. Influence of potassium fertilizer and mulching on growth and yield of chilli (Capsicum annuum L.). Acta Horticulture, v.369, p.311-318,1994.

HOCHMUTH, G.J.; SHULER, K.D.; MITCHELL, R.L.; GILREATH, P.R. Nitrogen crop nutrient requirement demonstrations for mulched pepper in Florida. Proceedings Florida State of Horticultural Society, v.100, p.205-209, 1987.

HOCHMUTH, J.G.; SHULER, K.D.; GILREATH, P.R.; MITCHELL, R.L. Field testing of revised Mehlichl predicted potassium fertilizer recommendation for mulched pepper. Soil and Crop Science Society of Florida Annual Proceedings, v.47, p.30-35, 1988.

ILEY, J.R.; OZAKI, H.Y. Nitrogen-potash ratio study with plastic mulched pepper. Proceedings Florida State of Horticulture Society, v.79, p.211-216, 1966.

KNAVEL, D.E. The influences of nitrogen on pepper transplant growth and yielding of plants grown with different levels of soil nitrogen. Journal American Society for Horticultural Science, v.102, n.5, p.533-535, 1977.

LESKOVAR, D.I.; CANTLIFFE, D.J.; STOFFELLA, P.J. Pepper (Capsicum annuum L.) root growth and its relation to shoot growth in response to nitrogen. Journal of Horticultural Science, v. 64, n.6, p.711-716, 1989.

LOCASCIO, S.J.; ALLIGOOD, M.R. Nitrogen and potassium source and $\mathrm{N}$-rate for drip-irrigated pepper. Proceedings Florida State of Horticultural Society, v.105, p.323-325, 1992.

LOCASCIO, S.J.; FISKELL, J.G.A.; MARTIN, F.G. Responses of bell pepper to nitrogen sources. Journal of the American Society for Horticultural Science, v.106, p.628-632, 1981.

LOCASCIO, S.J.; FISKELL, J.G.A.; GRAETZ, P.A.; HAUCK, R.D. Nitrogen accumulation by pepper as influenced by mulch and time of fertilizer application. Journal American Society for Horticultural Science, v.110, p.315-318, 1985.
MALAVOLTA, E.; VITTI, G.C.; OLIVEIRA, S.A. de. Avaliação do estado nutricional de plantas: princípios e aplicações. 2.ed. Piracicaba: Potafos, 1997. 319p.

MANCHANDA, A.K.; SINGH, B. Effect on plant density and nitrogen on yield and quality of bell pepper (Capsicum annuum L.). Indian Journal of Horticulture, v.44, n.3-4, p.250-252, 1987.

MANCHANDA, A.K.; SINGH, B. Effect of plant density and nitrogen on growth and fruit yield of bell pepper (Capsicum annuum L.). Indian Journal of Agronomy, v.33, n.4, p.445-447, 1988.

MARSCHNER, H. Mineral nutrition of higher plants. London: Academic Press, 1985.

MILLER, C.H.; McCOLLUM, R.E.; CLAIMON, S. Relationships between growth of bell peppers (Capsicum annuum L.) and nutrient accumulation during ontogeny in field environments. Journal American Society of Horticultural Science, v.104, n.6, p.852-857, 1979.

MISHRIKY, J.F.; ALPHONSE, M. Effect of nitrogen and plant spacing on growth, yield and fruit mineral composition of pepper (Capsicum annuum L.). Bulletin of Faculty of Agriculture University of Cairo, v.45, n.2, p.413-431, 1994.

MORENO, D.A.; PULGAR, G.; VÍLLORA, G.; ROMERO, L. Effect of $N$ and $K$ on fruit production and leaf levels of $\mathrm{Fe}, \mathrm{Mn}, \mathrm{Zn}, \mathrm{Cu}$ and $B$ and their biochemical indicator in capsicum plants. Phyton, v.59, n.1-2, p.1-12, 1996.

NAGAI, H. Pimentão. In: FAHL, J.I.; CAMARGO, M.B.P. de; PIZZINATTO, M.A.; FURLANI, A.M.C.; BETTI, J.A.; MELLO, A.M.T. de; MARIA, I.C. de (Ed.). Instruções agrícolas para o Estado de São Paulo. 6.ed. Campinas: IAC, 1995. p.335-337. (Boletim, 200).

O'SULLIVAN, J. Response of peppers to irrigation and nitrogen. Canadian Journal of Plant Science, v.59, p.1085-1091, 1979.

OLSEN, J.K.; LYONS, P.J.; KELLY, M. Nitrogen uptake and utilization by bell pepper in subtropical Australia. Journal of Plant Nutrition, v.16, n.1, p.177-193, 1993.

OLSEN, J.K.; LYONS, P.J. Petiole sap nitrate is better than total nitrogen in dried leaf for indicating nitrogen status and yield responsiveness of capsicum in subtropical Australia. Australian Journal of Experimental Agriculture, v.34, p.835-843, 1994.

OZAKI, H.Y.; OZAKI, C.T.; HAMILTON, M.G. The effects of applied nitrogen, phosphorus and potash on yield and growth of peppers. Proceedings Florida State of Horticultural Society, v.68, p.230-233, 1955. 
PEREIRA, E.C. Avaliação do crescimento e produtividade de pimentão matriciais de água no solo, em condições de casa de vegetação. Botucatu, 1995. 64p. Dissertação (Mestrado) - Faculdade de Ciências Agronômicas, Universidade Estadual Paulista.

POBLETE, E.R. El cultivo de las chiles dulces. Novedades Horticolas, v.16, n.1-4, p.21-27, 1971.

RAIJ, B. van; CANTARELLA, H.; QUAGGIO, J.A.; FURLANI, A.M.C. Recomendação de adubação e calagem para o Estado de São Paulo. Campinas: IAC , 1996. 285p.

RINCON, L. SAEZ, J,; BALSALOBRE, E.; PELLICER, C. Crescimiento y absorcion de nutrientes del pimiento grueso en cultivo bajo invernadero. Investigation Agraria: Produccion y Proteccion Vegetale, v.10, n.1, p.47-49, 1995.

SHUKLA, V.; SRINIVAS, K.; PRABHAKAR, B.S. Response of bell pepper to nitrogen, phosphorus and potassium fertilization. Indian Journal of Horticulture, v.44, n.1-2, p.81-84, 1987.

SINGH, K.; SRIVASTAVA, B.K. Effect of various levels of nitrogen and phosphorus on growth and yield of chilli (Capsicum annuum L.). Indian Journal of Horticulture, v. 45, p.319-324, 1988.
SINGH, V.; SHARMA, D.K.; BHAGWAN, B.V.K. Effect of level of nitrogen and time of its application on growth, yield and net-profit of chilli. Progressive Horticulture, v. 20, n.1-2, p.80-86, 1988.

SRINIVAS, K.; PRABHAKAR, B.S. Response of capsicum to nitrogen fertilization. Vegetable Science, v.9, p.71-74, 1982.

SUBHANI, P.M.; RAVISANKAR, C.; NARAYANA, N. Effect of graded levels and time of application of $\mathrm{N}$ and $\mathrm{K}_{2} \mathrm{O}$ on flowering, fruiting and yield of irrigated chilli. Indian Cocoa - Arecanut and Spices Journal, v.14, n.2, p.70-73, 1990.

VANANGAMUDI, K.; SUBRAMANIAN, K.S.; BASKARAN, M. Influence of irrigation and nitrogen on the yield and quality of chilli fruit and seed. Seed Research, v.18, n.2, p.114-116, 1990.

WOSTEN, J.H.M.; GENUCHTEN, M. TH. van. Using texture and other soil properties to predict the unsaturated soil hydraulic functions. Soil Science Society of America Journal, v.52, p.1762-1770, 1988.

Recebido para publicação em 22.10.98

Aceito para publicação em 26.07.99 